\title{
Vehicle monitoring under Vehicular Ad-Hoc Networks (VANET) parameters employing illumination invariant correlation filters for Pakistan motorway police
}

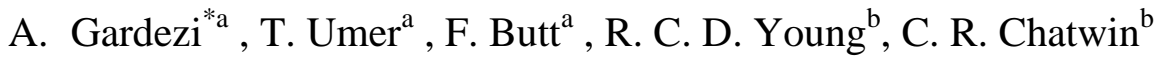 \\ ${ }^{a}$ Department of Computer Sciences, COMSATS Institute of Information Technology, Wah , Pakistan \\ ; ${ }^{b}$ Department of Engineering and Design,University of Sussex,Brighton, BN1 9QT ,United Kingdom
}

\begin{abstract}
A spatial domain optimal trade-off Maximum Average Correlation Height (SPOT-MACH) filter has been previously developed and shown to have advantages over frequency domain implementations in that it can be made locally adaptive to spatial variations in the input image background clutter and normalised for local intensity changes. The main concern for using SPOT-MACH is its computationally intensive nature. However in the past enhancements techniques were proposed for SPOT-MACH to make its execution time comparable to its frequency domain counterpart. In this paper a novel approach is discussed which uses VANET parameters coupled with SPOT-MACH in order to minimise the extensive processing of the large video dataset acquired from the Pakistan motorways surveillance system. The use of VANET parameters gives us an estimation criterion of the flow of traffic on Pakistan motorway network and acts as a precursor to the training algorithm. The use of VANET in this scenario would contribute heavily towards the computational complexity minimization of the proposed monitoring system.
\end{abstract}

Keywords: MACH filter, VANET, correlation filter, invariant pattern recognition

\section{Introduction}

The major aim of the OT-MACH filter is to find an optimal compromise between good discrimination ability and distortion tolerance in the presence of noise [1]. The MACH filter maximises the relative height of the average correlation peak with respect to the expected distortions. However, the peak height of the MACH filter is unconstrained, making it more difficult to interpret the results of the correlation. The $\mathrm{MACH}$ filter is derived by maximising a performance metric called the Average correlation height $(A C H)$. In addition, several other performance measures need to be balanced for better adaptability with different application scenarios. These measures are the Average Correlation Energy ( $A C E$ ), Average Similarity Measure ( $A S M$ ) and Output Noise Variance ( $O N V$ ) [1] , [2].

From equation (1) the resulting transfer function for an OT MACH filter (in the frequency domain) can be seen to be [1]:

$$
h=\frac{m_{x}^{*}}{\alpha C+\beta D_{x}+\gamma S_{x}}
$$

Included in the OT-MACH filter transfer function are: the terms $\alpha, \beta$ and $\gamma$, which are the non-negative weighting parameters; $m_{x}$ which is the average of the training image vector $x_{1}, x_{2}, \ldots, x_{N}$ (in the frequency domain); $C$ which is

*A.gardezi@ ciitwah.edu.pk ; Phone +92 51 9247000-3 
the diagonal power spectral density matrix of additive input noise and is usually set as the white noise covariance matrix, $C=\sigma^{2} I ; D_{x}$ is the diagonal average power spectral density of the training images [3].

Previous research has shown that when employing a frequency domain Optimal Trade-off Maximum Average Correlation Height (OT-MACH) filter degradation in performance could be seen when correlations were performed between a uniformly illuminated training dataset and a series of targets having variable illuminations [4].

Previously a moving window was used to implement a SPOT-MACH filter which can be locally modified depending upon its position in the input frame. This enables adaptation of the filter dependant on locally variant background clutter conditions and also enables the normalisation of the filter energy levels at each step. Thus the spatial domain SPOT-MACH filter offers an advantage over its frequency domain implementation as shift invariance is not imposed upon it. The only drawback of the spatial domain implementation is the amount of computational resources that are required for its real time implementation. In the past an optical correlator using a scanning holographic memory was proposed by Birch et al for the real time implementation of space variant filters of this type [7].

In order to formulate the transfer function for the SPOT-MACH filter, first the OT-MACH transfer function should be computed in the frequency domain as given by Equation (1). After considering the above mentioned factors, the SPOT$\mathrm{MACH}$ filter is then implemented in the spatial domain using a moving kernel to detect an object in a cluttered background plane of size, typically, between $512 \times 512$ and 1024x1024 pixels.

Normally a white noise covariance matrix is used as additive noise but in this case in order to counter the varying illumination changes, the maximum of the power spectral density of the input image is employed as the constant noise weighting.

The reference image spectrum is inverse Fourier transformed to yield a space domain image as shown in Equation (2):

$$
h^{*}=\operatorname{IFFT}\left[\frac{m_{x}^{*}}{\sqrt{\alpha C+\beta D_{x}+\gamma S_{x}}}\right]
$$

This array is truncated to the target object size which is, typically, less than 256x256 pixels. In addition, care must be taken to store the image as a bi-polar array (rather than an intensity image). The square root operation in the denominator of Equation (2) is required because the filtering operation must also be applied to the input image separately so this is preprocessed with the SPOT-MACH transfer function prior to its spatial domain correlation with the SPOT-MACH kernel function. The use of a moving kernel enables the filter to be adaptive to small areas of the image and hence minimise the impact of varying illumination patterns. Once the images have been passed through a SPOT-transfer function they are correlated using a spatial correlator. In this approach a windowing kernel normally the size of the target object is extracted from the composite image created by the SPOT-MACH and is scanned through the target image pixel by pixel. This enables adaptation of the filter dependant on background illumination variations and also enables the normalisation of the filter energy levels. The kernel can be normalised to remove a non-uniform brightness distribution if this occurs in different regions of the image. The main constraint in this implementation is the dependence on computational ability of the system.

In order to overcome this problem a spatial domain approach is proposed for the implementation of the OT-MACH filter which employs local window energy normalisation for the filter to facilitate more tolerance to illumination changes.

In this paper in order to effectively utilize the illumination invariance capabilities of the SPOT-MACH filter it was coupled with VANET over the Pakistan motorways network. The VANET provides the advantage of predicting the locations where a traffic congestion is about to occur. The Pakistan motorway network is a 600 mile stretch of roads through varying terrains ranging from mountainous to desert conditions. The major concern of the motorway police is to monitor un authorized vehicles over the roads in order to safe guard the security of the road users. Hence it is proposed that it is easier to detect an unauthorized vehicle where there is traffic congestion owing to the slow speed of the vehicles. 


\section{VANET Overview}

The VANET is a technology that uses moving vehicles as inter-connecting nodes in a network to create a mobile network. In VANET every vehicle is a node hence when within the proximity of 100-300 meters each node establishes a link in turn creating a wide area network. When a vehicle is outside the range of the network the associated node is removed. The removed node is replaced by other vehicles joining the range of the network; the interconnection of nodes creates an intervehicle channel. In the recent years the applications of VANET have increased widespread ranging from Post Crash Notification (PCN), Road Hazard Control Notification, Slow/Stop Vehicle Advisor, and Congested Road Notification $(\mathrm{CRN})$. Also being explored is the aspect of using VANET for social networking for the purpose of users sharing information with each other over a specific route like informing about hazardous areas in advance [7].

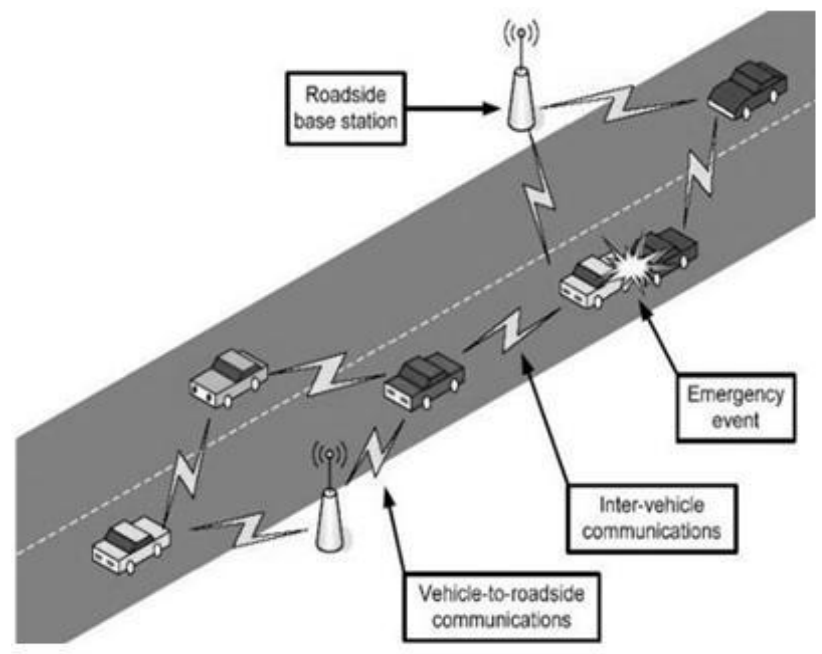

Figure 1. A simple VANET system [7]

A simple VANET system presented by Figure 1, it can be seen that there are two types of communications taking place, vehicle to vehicle (V2V) and Vehicle to Roadside (V2R). The V2R model is commonly used to develop a large network which uses a dedicated frequency range allocated for VANET [7].

Using parameters such as speed, density flow, safety distance and length the traffic engineers have defined different traffic flow models [8]. These models are based on both microscopic and macroscopic parameters to implement traffic codes. In this paper using the above mentioned parameters have been used to analyse the road conditions and vehicles characteristics within a specific area of interest i.e. M1 motorway Islamabad to Lahore, Pakistan.

The design and implementation of vehicular ad-hoc depends on the prevailing road conditions. The vehicular characteristics of moving traffic under diverse road conditions are used by research studies to focus the key issues of VANET. The use of density dynamics for the assignment of dynamic transmission range in vehicular ad-hoc network was performed in these studies $[9,10]$. The mobility of vehicles on the road is also focused to enhance the performance of VANET.

The available models of traffic flow theory such as fluid dynamic model, car following model [5] are used for representing traffic flow in many studies for VANET. In [11], analytical modelling of VANET by considering the traffic flow models and analysing the statistical properties of connectivity is well elaborated. The use of queuing theory and consideration of road traffic parameters for connectivity analysis are discussed and presented as analytical model for connectivity in [12].

In this paper a traffic flow model was simulated based on the data gathered from the Pakistan motorway police. Since establishing a VANET requires extensive infrastructure and development costs, hence it is difficult to implement in developing countries. 
In the case of Pakistan motorway police although a conventional VANET infrastructure doesn't exist, but is in the pipeline to be deployed in the near future. In the absence of VANET infrastructure and to propose its usefulness the data was acquired from the electronic tag system used for toll collection on motorway. This electronic system acquires information from each car as it passes through the toll and hence gives an estimation how many cars are present on the motorway at any instance. The toll booths are present at each junction of the motorway, and hence data can be updated for vehicles entering or leaving the motorway. A working toll booth at the Islamabad to Lahore motorway is given by Figure 2 below.

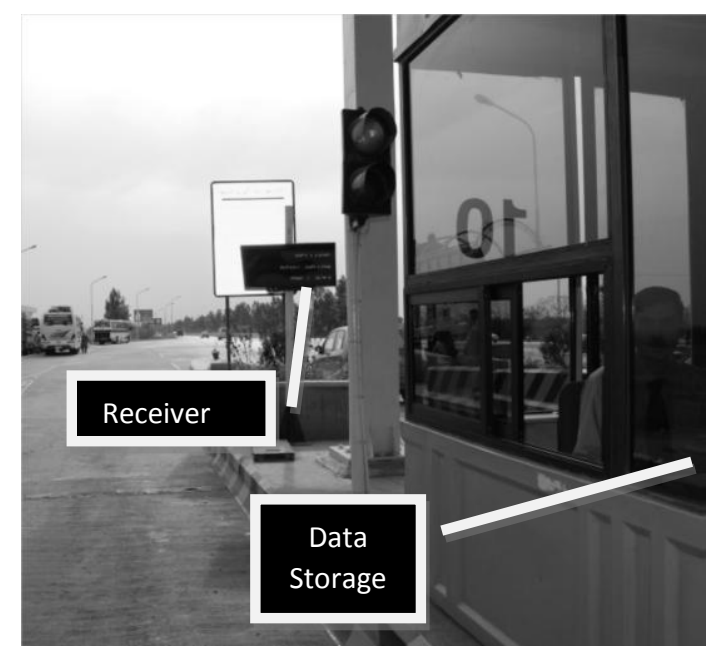

Figure 2. Pakistan motorway M1 toll booth with electronic tag system

A simulation model was generated using a VANET simulator to predict areas where congestion might occur and to focus the SPOT-MACH in those areas only to minimise computations. There are different models in VANET that can be used to predict the behaviour of traffic movements, for this paper a deterministic fluid dynamic model was considered to predict the density estimation of traffic in a specific area.

\section{Operation of SPOT-MACH filter}

The SPOT-MACH transfer function given by equation 2 creates a spatial domain composite image of multiple orientations that can be effectively used as a kernel to perform the normalized correlation with the target scene.A further point to be noted in the case of the SPOT-MACH filter is the size of the correlation output plane which is smaller as compared to the frequency domain plane. This is due to the fact that the windowing function is used which will effectively return a lesser number of correlation points if zero padding is not used. The correct size of the correlation plane can be established from:

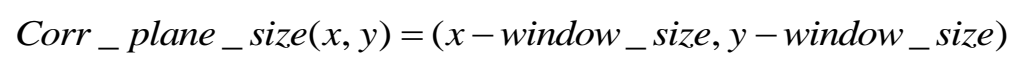

Since the correlation output plane (COP) size has been compensated, the location of the peak can be made to correspond to the location of the object in the input scene. Thus in order to determine the corrected location of the target object, the transformation given by Equation 4 should be applied to the peak coordinates of the SPOT-MACH filter:

$$
\text { Corr_Outputplane }=\left(x-\frac{\text { winsize }}{2}, y-\frac{\text { winsize }}{2}\right)
$$

Having established that the SPOT-MACH filter is able to detect similar shaped objects, its capabilities were tested using the same dataset acquired from the Pakistan motorway police. The main aim of this test was to assess the tolerance of the SPOT-MACH filter to the change in illumination when false targets can be present on the motorway. 
The SPOT-MACH was tested on a slightly modified criterion; the aim was to detect large vehicles. As Pakistan motorway operates on very strict heavy traffic rules, hence there are certain timings allowed for large vehicles. In order to test the capabilities of the filter in different conditions three sets of scenes were acquired from the overhead bridge cameras. The target scenes are given by Figure 3 below:

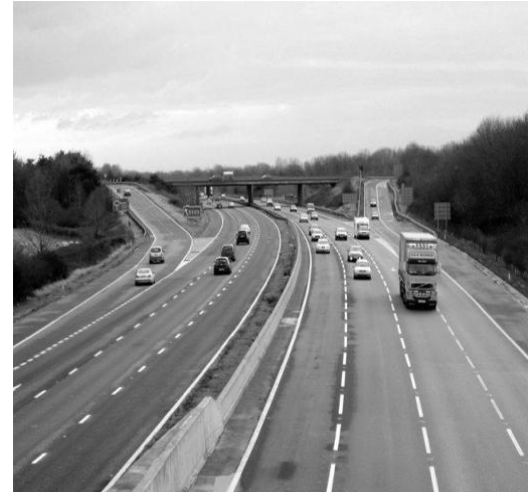

a

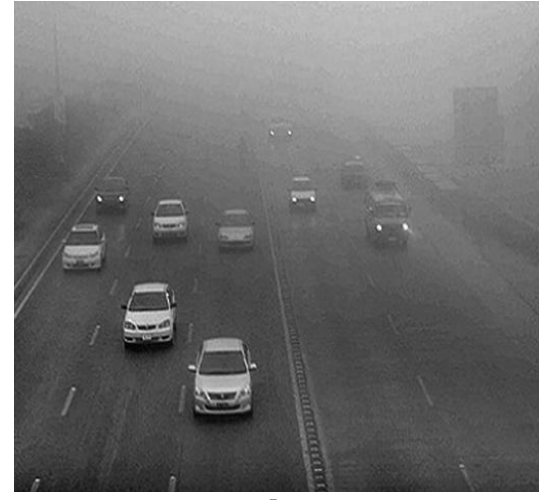

b

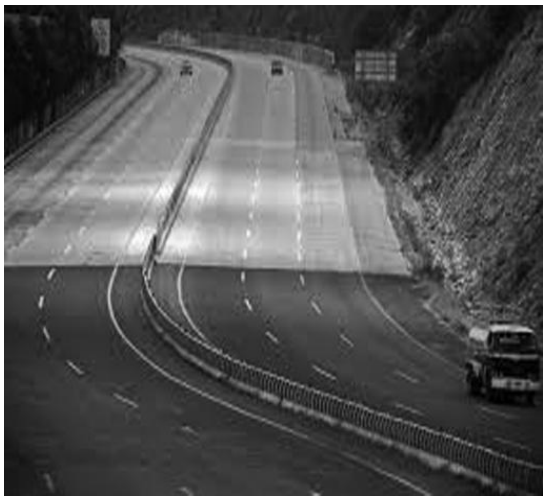

Figure 3. a) Motorway in Uniform Brightness , b) Motorway in foggy condition c) Motorway in non-uniform brightness

The Figure 3 presents three scenarios, where a) shows the motorway in day light, b) shows the motorway in foggy conditions with extremely poor lighting conditions and c)shows the condition in non-uniform brightness where the shadow of the mountain is over the target object.

As discussed earlier the SPOT-MACH filter was tuned to detect the shape of large targets by setting the parameters to $\alpha=0.001, \beta=0.7, \gamma=0.0001$.

The reference image used for the training filter is given by Figure 4 below.

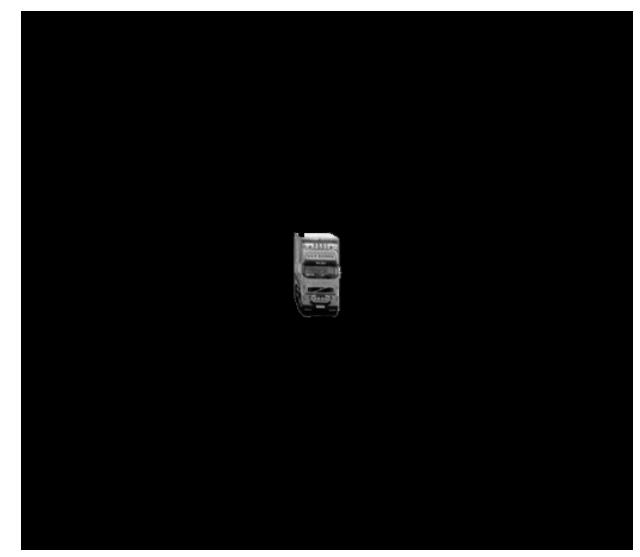

Figure 4. Reference Image for SPOT-MACH filter

Although the SPOT-MACH filter has the ability to be trained for multiple orientations for these tests only a single orientation of 10 degrees was considered. This was done intentionally to restrict the detection abilities of the filter, as it was used to identify the size of the vehicles only. 
The correlation output plane for the SPOT-MACH filter when Figure 4 was used as the reference image and the Figure 3 as the target is given by Figure 5 below.
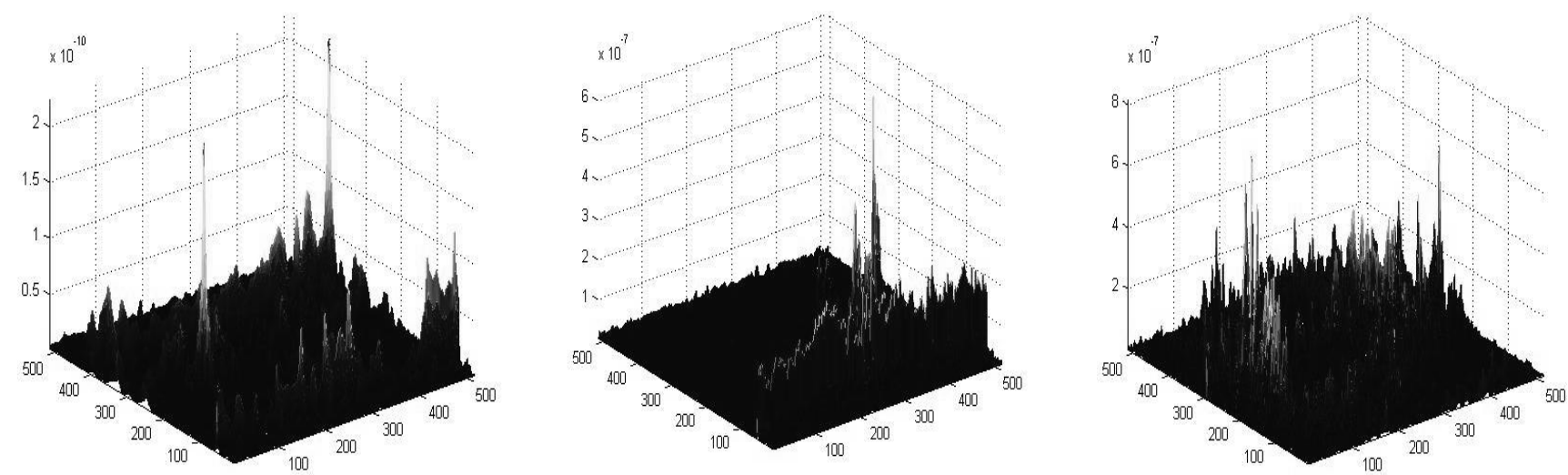

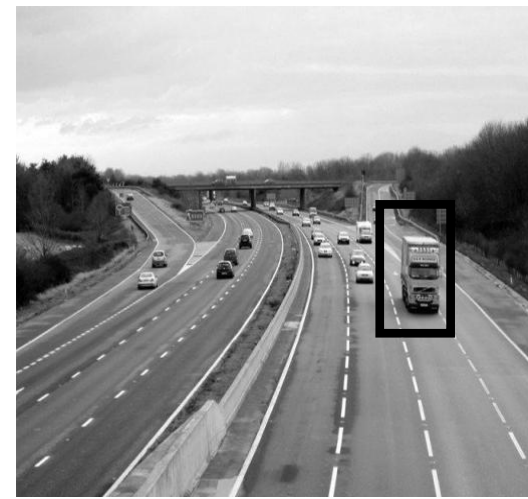

COP for Figure $3 \mathrm{a}$

a

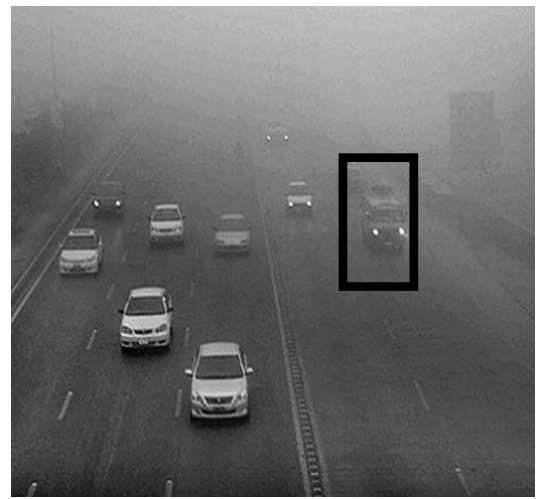

COP for Figure 3b

b

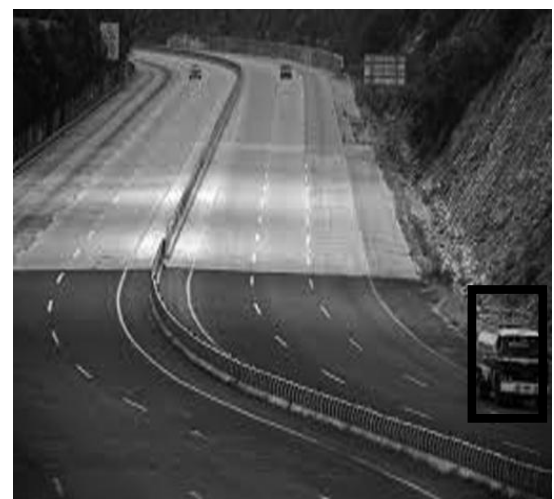

COP for Figure 3c

c

Figure 5. a) Uniform Brightness results, b) Foggy weather low light results, c) Non-uniform brightness results

The correlation output plane from the images acquired from the motorway cameras are presented above. From Figure 5a it can be seen that the filter has identified the large vehicle from the target image but there was also a false detection from the edges of the motorway. In Figure $5 \mathrm{~b}$ it can be seen that although there were very poor lighting conditions the filter manages to identify the target object. In Figure $5 \mathrm{c}$ it can be seen that the filter fails to correctly identify the target object, but this was due to the presence of a very large illumination gradient and high energy concentration in the edges. Although the filter picks up the object the false detections could have been minimised if more orientations were added in the training image.

Now it is evident that the SPOT-MACH filter is able to detect the desired objects from the target imagery, the next step is to minimise the computational requirements. It is not possible to monitor the data acquired from the motorway cameras in real time owing to infrastructure constraints of the Pakistan motorway police. A technique is proposed in the next session which couples VANET with SPOT-MACH to predict the concentration of traffic on the motorway hence enabling the filter to target a specific area and time. 


\section{Fluid Dynamic Model for Density estimation of Heterogeneous Traffic Flow}

As discussed above the road traffic is characterized as fluid flow by the traffic engineers. This fluid like behaviour of moving vehicles on the road leads to represent different traffic models known as continuum traffic flow models. These models tend to possess the characteristics of fluid dynamics which represents traffic flow in the form of the conservation law. It provides the traffic flow and density as a function of time and space. It relates the behaviour of traffic in the form of partial differential to represent parameters like flow, speed and density.

The model presented in [13], considered a heterogeneous traffic flow in a single-lane, one way, and semi-infinite highway environment. The location space is characterized with the interval $[0, \infty]$ the starting of the road is marked by boundary point 0 , which is consider as spatial origin. The road is divided into segments represented by $\mathrm{r}=1$, 2, 3, 4, due to road intersections. The vehicles can join or leave the moving stream at these intersections called junctions. For the first segment of the road, the number of arrivals of all type of vehicles up to time $t$, is counted by an arrival process $\{\mathrm{G}(\mathrm{t}) \mid-\infty<\mathrm{t}<\infty\}$, which is assumed to be finite with a probability of 1 . This arrival process is characterized by external arrival rate function for all type of vehicles ' $\lambda(t)$ ' which is non-negative and integrable.

Two types of vehicles were considered car and a bus. So the arrival rate for cars and busses is given by external arrival rate function ' $\lambda_{c}(t)$ ' and ' $\lambda_{b}(t)$ '.

The conservation equation relating important parameters of traffic flow is given as [12],[14]:

$e^{+}(x, t)=n(x, t)+f(x, t)+e^{-}(x, t)$

Where $n(x, t)$ is the total number of vehicles in location $(0, x)$,

$f(x, t)$ is the number of vehicles passing past position $x$,

$e^{+}(x, t), e \quad(x, t)$ are vehicles arriving and departing rate.

The conservation equation when considering cars and buses is given below [12].[14].

$$
\begin{aligned}
& e_{c}{ }^{+}(x, t)=n_{c}(x, t)+f_{c}(x, t)+e_{c}(x, t) \\
& e_{b}{ }^{+}(x, t)=n_{b}(x, t)+f_{b}(x, t)+e_{b}(x, t)
\end{aligned}
$$

For having partial differential equation form of conservation equation relating density, flow, arrival and leaving rate. We are differentiating equation $6 \mathrm{a}$ and $6 \mathrm{~b}$ by time and space [9],[13]. Using the operator $\frac{\partial^{2}}{\partial x \partial t}$ on the equation 6a and 6b.

$$
\begin{gathered}
\frac{\partial n_{c}(x, t)}{\partial t}+\frac{\partial f_{c}(x, t)}{\partial x}=e_{c}(x, t)-e_{c}(x, t) \\
\frac{\partial n_{b}(x, t)}{\partial t}+\frac{\partial f_{b}(x, t)}{\partial x}=e_{b}(x, t)-e_{b}(x, t)
\end{gathered}
$$

According to the fundamentals relation of traffic flow theory[14].

$$
f(x, t)=n(x, t) * u(x, t)
$$

Using equation $(6(a, b))$ and $(7(a, b))$ we have

$$
\frac{\partial n_{c}(x, t)}{\partial t}+\frac{\partial\left[\mathbf{n}_{c}(x, t) u_{c}(x, t)\right]}{\partial x}=e_{c}^{+}(x, t)-e_{c}{ }^{-}(x, t)
$$




$$
\frac{\partial n_{c}(x, t)}{\partial t}+\frac{\partial\left[\mathbf{n}_{c}(x, t) u_{c}(x, t)\right]}{\partial x}=e_{b}{ }^{+}(x, t)-e_{b}{ }^{-}(x, t)
$$

These relations formed the one dimensional version of generalized conservation law for fluid motion in partial differential form representing cars and busses in heterogeneous traffic flow. By applying chain rule and defining velocity as [13]:

$u(x(t), t)=\frac{d x(t)}{d t}$

After substituting the values from equation 5 we get equation for finding density of two different type of vehicle.

$$
\begin{aligned}
& \frac{d n_{c}(x(t), t)}{d t}=e_{c}{ }^{+}(\mathrm{x}, \mathrm{t})-e_{c}(\mathrm{x}, \mathrm{t})-\frac{\partial \mathrm{u}_{\mathrm{c}}(\mathrm{x}, \mathrm{t})}{\partial x} \cdot \mathrm{n}_{\mathrm{c}}(\mathrm{x}(\mathrm{t}), \mathrm{t}) \\
& \frac{d n_{b}(x(t), t)}{d t}=e_{b}{ }^{+}(\mathrm{x}, \mathrm{t})-e_{c}(\mathrm{x}, \mathrm{t})-\frac{\partial \mathrm{u}_{\mathrm{b}}(\mathrm{x}, \mathrm{t})}{\partial x} \cdot \mathrm{n}_{\mathrm{b}}(\mathrm{x}(\mathrm{t}), \mathrm{t}) \\
& N(x, t)=n_{c}(\mathrm{x}(\mathrm{t}), \mathrm{t})-\mathbf{n}_{\mathrm{b}}(\mathrm{x}(\mathrm{t}), \mathrm{t})
\end{aligned}
$$

The equations $10 \mathrm{a}$ and $10 \mathrm{~b}$ have the effect of microscopic variables of headway and safety distance for different type of vehicles in the traffic stream and can be applied for finding total vehicle density with the use of equation.11 having effect of dynamic road conditions [10],[11] and [12].

\subsection{Stochastic model for heterogeneous traffic flow}

The density estimation from fluid dynamics model provides the expected number of vehicles. To capture actual number of vehicles within the maximum travelled area, but still we need additional distribution information from the stochastic model. In stochastic model the density and flow rate for car and bus will be defined as partial derivate of expected values of density and traffic flow [11],[15] and [16].

$$
\begin{aligned}
& n_{c}(\mathrm{x}, \mathrm{t})=\frac{\partial E\left[\partial N_{c}(x, t)\right]}{\partial x} \\
& n_{b}(\mathrm{x}, \mathrm{t})=\frac{\partial E\left[\partial N_{b}(x, t)\right]}{\partial x} \\
& f_{c}(\mathrm{x}, \mathrm{t})=\frac{\partial E\left[\partial F_{C}(x, t)\right]}{\partial x} \\
& f_{b}(\mathrm{x}, \mathrm{t})=\frac{\partial E\left[\partial F_{b}(x, t)\right]}{\partial x}
\end{aligned}
$$

Where $\boldsymbol{n}_{c}, \boldsymbol{n}_{b}$ are density rate $\& f_{c}$ and $f_{b}$ are flow rates.

Under the implementation of Poisson Arrival Location Model (PALM), the arrival process $\left\{\mathrm{G}_{\mathrm{c}}(\mathrm{t}), \mathrm{G}_{\mathrm{b}}(\mathrm{t}) \mid-\infty<\mathrm{t}<\infty\right\}$ for car and bus for the first segment of road is a non-homogenous Poisson process with non-negative and integratable external arrival rate function $\lambda_{c}(t)$ for car and $\lambda_{b}(t)$ for bus respectively [13]. So the number of arrivals in the interval (t1, $\left.t 2\right)$ is Poisson with mean.

$$
\begin{aligned}
& \int_{t_{1}}^{t_{2}} \lambda_{c}(w) d(w) \\
& \int_{t_{1}}^{t_{2}} \lambda_{b}(w) d(w)
\end{aligned}
$$


According to [12] we can have, the total random number of all type of vehicles within the range (o, $x)$ at time ' $t$ ', via stochastic integration starting with Poisson process $\left(G_{c}, G_{b}\right)$ where $\left(G_{c}(t), G_{b}(t)\right)$ counts the number of arriving cars and buses to the road segment up to the time t.

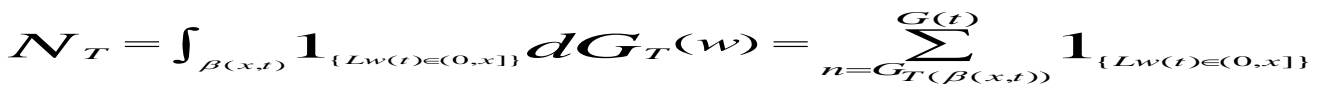

Hence for all real $\mathrm{t},\{\mathrm{NT}(\mathrm{x}, \mathrm{t}) \mid \mathrm{x} \geq 0\}$ is a Poisson process with

$$
E\left[N_{T}(x, t)\right]=\int_{B(x, t)}^{t} \alpha(w) d(w)
$$

Where $G_{r}$ is the rth jump time of $G$, counting backward from time t. 1c is an indicator function which that turns 1 if $C$ is true and 0 otherwise. $L_{w(t)}$ is the location process, which defines the position of vehicle on the road segment at time $t$ arrived at time $\mathrm{W}$. Let $\beta(\mathrm{x}, \mathrm{t})$ denote the road entrance time for any type of vehicle to be at position $\mathrm{x}$ by the time $\mathrm{t}$. for the other vehicles arriving after $\beta(\mathrm{x}, \mathrm{t})$ they will be still in position $\mathrm{x}$ at time $\mathrm{t}$. Using the models considered above a traffic model for motorway traffic was proposed in the forthcoming section.

\section{Proposed VANET model}

In the proposed model we considered heterogeneous traffic flow in a single-lane, one way, semi-infinite highway environment. The road is divided into number of road segments represented by $r=1,2,3,4$...., representing the highway interchanges.

It was assumed that no vehicle is joining or leaving the road at intersections. The vehicles arrive only at location 0 at a constant rate $\lambda(t)=20$ vehicles/min this includes all type of vehicles.

For the heterogeneous traffic flow the arrival rate for cars and buses is defined as ' $\lambda_{c}(t)$ ' and ' $\lambda_{b}(t)$ ' having different arriving ratios for different road scenarios.

It was considered that initially there are no vehicles on the road when traffic starts. So $n_{c}(x, 0)=0 \& n_{b}(x, 0)=0$ for all $x$ belongs to $X$ where $X$ is location space in $\mathrm{km}$.

The initial velocity for all vehicles as calculated from car following model speed equation from [13] at $(\mathrm{t}=0)$ will be the mean free speed $V_{f}=1 \mathrm{~km} / \mathrm{min}$

On the road at the distance of $4 \mathrm{~km}$, a road incident was introduced to capture the effect of vehicles interaction due to the safety distance and front density of the road traffic on vehicular density.

During the traffic stoppage period due to road incident traffic is stopped for 4 to $4.5 \mathrm{~min}$ for $30 \mathrm{sec}$.

For the implementation of road junction extra $0.012 \mathrm{~km}$ distance is consider before the incident.

The traffic condition on the road is dynamic due to the changing arriving ratio for different type of vehicles. Due to these arriving patterns the safety distance between the vehicles is also affected. The results from the proposed VANET model simulation are given in the forthcoming section.

\subsection{Results and Analysis}

In Figure 6, to capture the effect of heterogeneousness traffic flow on density for cars and buses, initially 20 vehicles are considered. The external arrival rat is categorized for two types of vehicles car and a bus given as:

$\operatorname{Car}\left(\lambda_{\mathrm{c}}(\mathrm{t})\right)=12 / 20$

$\operatorname{Bus}\left(\lambda_{\mathrm{b}}(\mathrm{t})\right)=08 / 20$. 
The safety distance between the cars and busses is considered as

i.) $\quad 2 *$ car-length for cars

ii.) The safety distance for busses is fixed as $0.012 \mathrm{~km}$.

As the traffic flow on the road the velocity profile is built under the influence of safety distance and front density of road traffic. The calculated velocity is affecting the road density due to iterative process as it is used by the differential equation for density estimation in equation 7(a) and 7(b).

At the time $4 \mathrm{~min}$ due to the road incident the vehicle platoon formation is created which lasts for stopping period shows at time $4.5 \mathrm{~min}$ in the graph, as the vehicles stoppage goes away the vehicles disperse and density curve shows normal behaviour after time $5 \mathrm{~min}$.
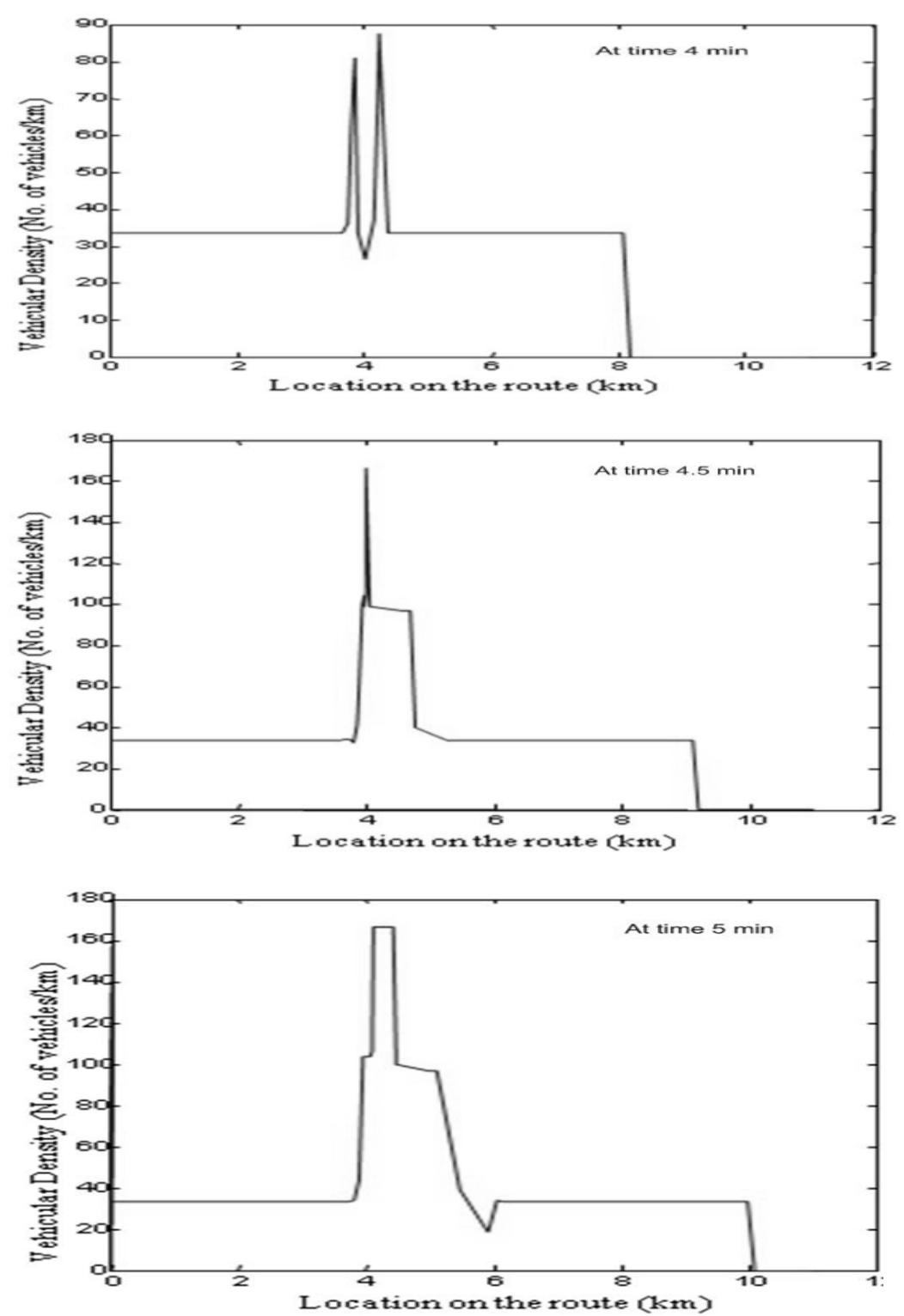

Figure 6. Proposed VANET model simulation to show the flow of traffic
- $\quad$ The VANET model shows that an incident has occurred at time $=4$ minutes and distance of $4 \mathrm{~km}$ from the observation point.

- VANET shows traffic built up from 35 to 90 vehicles.

- $\quad$ The affected route area is around $1 \mathrm{~km}$.

- After 4.5 minutes the VANET model shows the traffic built up has exceeded to 180 vehicles.

- The exponential decrease in graph shows the dispersion of the traffic built up.

- The affected route area is also decreased.

- After 5 minutes the VANET model shows the traffic built up has exceeded to 180 vehicles.

- The exponential decrease in graph shows the dispersion of the traffic built up. 
From Figure 6 the VANET simulation of the proposed model shows that the traffic density and the affected area when a traffic built up occurs can be predicted.

This information can be used to optimize the working of the SPOT -MACH filter, as two types of external arrival rates were considered for car and a bus. It is possible for the VANET to predict a target area for the SPOT-MACH to concentrate. From the above model it was predicted that the maximum density of the vehicles would be in an area between 4-5 kilometers from the origin point. When a total area of 10 kilometers is being considered, the amount of computational complexity is reduced by $1 / 10^{\text {th }}$ when using the VANET model. The proposed model is further elaborated by Figure 7 given below:

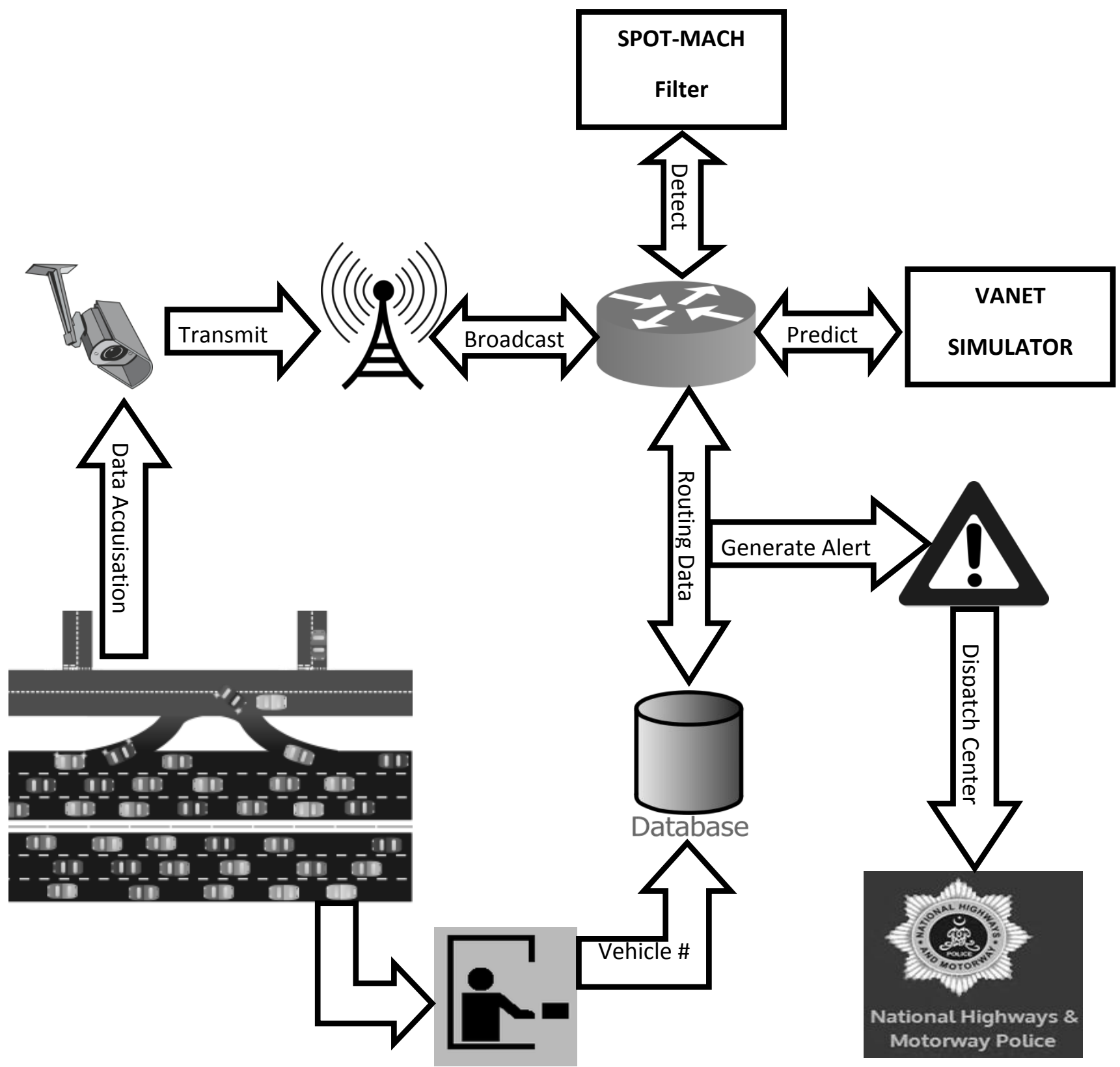

Figure 7. Motorway Police vehicle monitoring system using VANET and SPOT-MACH filter 
A Motorway Police vehicle monitoring system is proposed in Figure 7, the salient features of this system are as follow:

i.) The cameras acquire the motorway traffic video and transmit it to the central database.

ii.) The electronic tagging booths at entry and exit points store the vehicle information and transmit it to the central database.

iii.) The VANET simulator acquires the information from the central database and predicts the occurrence of an incident.

iv.) The SPOT-MACH filter uses the predicted information from VANET to detect target vehicles from the predicted parameters only.

v.) If a target is detected, a alarm flag is raised which alerts the motorway police dispatch center by sending the location.

vi.) The proposed system saves computational time by sending the data from the predicted time frames only to the SPOT-MACH filter.

From Figure 7 it can be seen that by coupling VANET with SPOT-MACH an optimized can be created for the effective detection of vehicles over a large terrain. As when a large terrain is considered the minimization of computations ensures real time processing of the acquired data.

\section{Conclusion}

One of the main advantages of the SPOT-MACH filter is the ability to allow localised normalisation of the filter which is not possible in the frequency domain implementation of the MACH filter. Results have been presented which indicate a spatial domain implementation of the filter is able to detect, locate and recognise a target object within a non-uniformly scene.

VANET was used as an optimization step to minimise the computational time required by the SPOT-MACH filter. Three Test cases were created, using visual band imagery, containing varying degrees of illumination non-uniformity. These scenes were used for a detailed comparison of the SPOT-MACH filter performance using VANET. A traffic model was created in VANET simulator which was used to predict the traffic congestion time and location when an incident occurs over a motorway. It was seen that that the amount of data was reduced to $1 / 10^{\text {th }}$ of its original size when VANET predictions were taken into account.

Hence using a novel approach by coupling VANET with correlation filters an effective security monitoring system can be created.

\section{References}

[1] Mahalonobis, A. , Kumar, B.V.K. , Song, S., Sims, S.R.F. , Epperson, J.F., "Unconstrained correlation filters,". Applied Optics 33 , 3751-3759(1994).

[2] Mahalonobis, A. , Kumar, B.V.K. , "Optimality of the maximum average correlation height filter for detection of target in noise," Optical Engineering 36(1), 2642-2648(1997).

[3] Gardezi, A. , Birch , P. , Young , R. and Chatwin, C. , " Implementation of the Maximum Average Correlation Height (MACH) filter in the spatial domain for object recognition from clutter backgrounds," Proc. SPIE 7696, (2010).

[4] Gardezi, A. , Al-Kandri, A. , Birch, P. , Young, R. and Chatwin, C. " A space variant maximum average correlation height (MACH) filter for object recognition in real time thermal images for security applications," Proc. SPIE 7838, (2010).

[5] Gardezi, A. , Al-Kandri, A. , Birch, P. , Young, R. and Chatwin, C. , " Enhancement of the speed of space-variant correlation filter implementations by using low-pass pre-filtering for kernel placement and applications to real-time security monitoring," Proc. SPIE 8055, (2011). 
[6] Birch, P., Gardezi, A. ,Mitra,B.K., Young, R. and Chatwin, C., "An optical space domain volume holographic correlator," Proc. SPIE 7340,(2009).

[7] Nesh, W., "Vehicular networking and its applications," HCLTECH, 7 March 2011,

http://www.hcltech.com/blogs/engineering-and-rd-services/vehicular-networking-and-its-applications (12 January 2016).

[8] Hoogendoorn, S. P. , Bovy, P. H. L. ,"State-of-the-Art of Vehicular Traffic Flow Modeling," J. Sys. and Control Eng., vol. 215, no. 4, 2001, pp. 283-304.

[9] Artimy, M. , Roberstson, W. , Philips, W. , “Assignment of dynamic transmission range based on estimation of vehicle density," in Proc. of the 2nd ACM int'l workshop on Vehicular ad hoc networks (VANET'05), New York, NY, USA, 2005, pp. $40-48$.

[10] Panichpapiboon, S. , Pattara-atikom, W. , "Evaluation of a neighbour based vehicle density estimation scheme," in Proc. IEEE Int. Conf. ITST, Phuket, Thailand, Oct. 2008, pp. 294-298.

[11] Khabazian, M. , Mehmet Ali, M. K. , "A Performance Modeling of Connectivity in Vehicular Ad Hoc Networks," IEEE Trans. Veh. Technol., vol.57, no. 4, pp.2440-2450, Jul.2008.

[12] Yousefi, S. , Altman, E. , El-Azouzi, R. , Fathy M. , “Analytical Model for Connectivity in Vehicular Ad Hoc Networks" IEEE Trans. Veh.Tech, vol. 57, no. 6, pp.3341-3356, Nov 2008.

[13] Umer,T., Zhiguo,Ding., Honary.B., Ahmad.H., "Connectivity Dynamics Under Microscopic Parameters in Signalized Heterogeneous Traffic Flow for VANET", The $12^{\text {th }}$ Annual Post Graduate symposium on the Coveragence of Telecommunication, Networking and Broadcasting, June 2012, pp.69-73.

[14] Leung, K. K. , Massey, W. A. , Whitt, W. ,"Traffic Models for Wireless Communication Networks," IEEE J-SAC, vol. 12, no. 8. 1994

[15] Massey, W. A. , Whitt, W. , “A Stochastic Model to Capture Space and Time Dynamics in Wireless Communication Systems,” Prob. Eng. Inf. Sci, vol.8, pp.541 - 569, 1994.

[16] Massey, W. A. , Whitt, W. , "Network of Infinite Server Queues with Non stationary Poisson Input," Queuing Syst., vol.13, pp.183 - 250, 1993. 